\title{
Synthesis and antimicrobial activity of new Schiff bases containing coumarin moiety and their spectral characterization
}

\author{
V. S. V. Satyanarayana, P. Sreevani, Amaravadi Sivakumar, and V. Vijayakumar* \\ Chemistry Division, School of Science and Humanities, VIT University, Vellore-632014, India \\ E-mail:kvpsvijayakumar@gmail.com
}

\begin{abstract}
New Schiff base 2-[(4-Methyl-2-oxo-2H-chromen-7-yl)oxy]-N'-(substitutedmethylene)aceto hydrazides (4a-p) were synthesized by the condensation of aryl/hetero aromatic aldehydes with 2-[(4-methyl-2-oxo-2H-chromen-7-yl)oxy]acetohydrazide under conventional and microwave conditions and characterized through IR, ${ }^{1} \mathrm{H}$ NMR and Mass spectral data. The synthesized compounds have been screened for antimicrobial activity.
\end{abstract}

Keywords: 2-[(4-Methyl-2-oxo-2H-chromen-7-yl)oxy]-N'-(substitutedmethylene)acetohydrazides, conventional method, microwave-irradiation, schiff base, spectral data, antimicrobial analysis

\section{Introduction}

Azomethine group $(-\mathrm{C}=\mathrm{N}-)$ containing compounds typically known as Schiff bases have been synthesized by the condensation of primary amines with active carbonyls. Schiff bases form a significant class of compounds in medicinal and pharmaceutical chemistry with several biological applications that include antibacterial, ${ }^{1-6}$ antifungal ${ }^{3-6}$ and antitumor activity. ${ }^{7-8}$ They have been studied extensively as a class of ligands ${ }^{9-11}$ and are known to coordinate with metal ions through the azomethine nitrogen atom.

Schiff base complexes play a vital role in designing metal complexes related to synthetic and natural oxygen carriers. ${ }^{12}$ Metal complexes make these compounds effective as stereospecific catalysts towards oxidation, reduction, hydrolysis, biological activity and other transformations of organic and inorganic chemistry. ${ }^{13}$ In organic compounds the presence of $-\mathrm{C}=\mathrm{N}-$ along with other functional groups form more stable complexes compared to compounds with only $-\mathrm{C}=\mathrm{N}-$ coordinating moiety.

Similarly coumarin derivatives have been of great interest because of their role in natural and synthetic organic chemistry. Many products which contain a coumarin subunit exhibit biological activity such as molluscicides ${ }_{14}^{14}$ anthelmintic, hypnotic, insecticida ${ }^{15}$ activity and some are serving as anticoagulant agents ${ }^{16}$ and fluorescent brighteners. So coumarins containing a Schiff base are expected to have enhanced antitumor and other biological activities. It is well 
established that the biological activity associated with the hydrazone compounds attributed to the presence of the active pharmacophore $(-\mathrm{CONH}-\mathrm{N}=\mathrm{C}-)$. Hence many hydrazone compounds containing this active moiety showed good anticancer bioactivities according to the literature. ${ }^{17}$

Microwave irradiation of organic reactions has rapidly gained popularity as it accelerates the reaction towards a variety of synthetic transformations, solventless procedures without the use of supporting reagents and hence eco-friendly. Chemical transformations that took hours or even days to complete can now be accomplished in minutes. Microwave energy offers numerous benefits for performing synthesis such as increased reaction rates, enhanced yields and cleaner chemistries. Hence, in this paper, we are reporting the synthesis of coumarin analogues with Schiff bases of 2-[(4-Methyl-2-oxo-2H-chromen-7-yl)oxy]acetohydrazide with different aryl and hetero aromatic aldehydes by conventional and microwave assisted methods, and their characterization through spectral data such as IR, ${ }^{1} \mathrm{H}$ NMR and Mass spectra. Their antimicrobial activity was also evaluated.

\section{Results and Discussion}

Resorcinol treated with ethylacetoacetate in the presence of sulphuric acid at $10{ }^{\circ} \mathrm{C}$ yielded 7Hydroxy-4-methyl-2H-chromen-2-one (1). ${ }^{18}$ Treating compound $\mathbf{1}$ in dry DMF with ethyl chloroacetate in the presence of $\mathrm{K}_{2} \mathrm{CO}_{3}$ at $80{ }^{\circ} \mathrm{C}$ for 10 hours yielded Ethyl 2-[(4-methyl-2-oxo$2 \mathrm{H}$-chromen-7-yl)oxy]acetate (2) in $82 \%$ yield, whereas in literature the conversion of $\mathbf{1}$ into 2 was reported under the refluxing conditions for $10 \mathrm{hrs}$ in dry acetone ${ }^{19-24}$ with $40 \%$ yield. Much variation in yield percentage was not observed though the conversion of $\mathbf{1}$ into $\mathbf{2}$ is also effected by using anhydrous sodium bismuthate as catalyst instead of $\mathrm{K}_{2} \mathrm{CO}_{3}$ (Scheme 1).
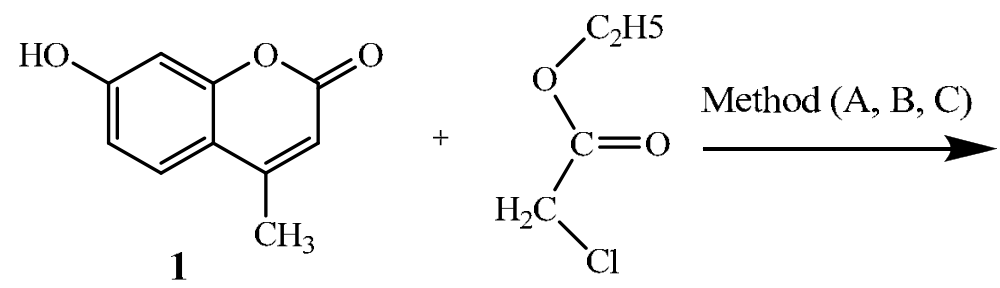<smiles>CCCCOC(=O)COc1ccc2c(C)cc(=O)oc2c1</smiles>

\section{Method A. Conventional using $\mathrm{K}_{2} \mathrm{CO}_{3}$ Method B. Conventional using $\mathrm{NaBiO}_{3}$ Method C. Solvent-free synthesis under microwave irradiation}

\section{Scheme 1}

Compound 2 was heated with hydrazine hydrate in ethanol on a water bath for 1 hour to obtain 2-[(4-Methyl-2-oxo-2H-chromen-7-yl)oxy]acetohydrazide (3). The 2-[(4-Methyl-2-oxo-2Hchromen-7-yl)oxy]-N'-(substitutedmethylene)acetohydrazides (4a-p) synthesized by the 
condensation of 2-[(4-Methyl-2-oxo-2H-chromen-7-yl)oxy]acetohydrazide (3) with different aryl/hetero aromatic aldehydes and characterized through the spectral data (Scheme 2).

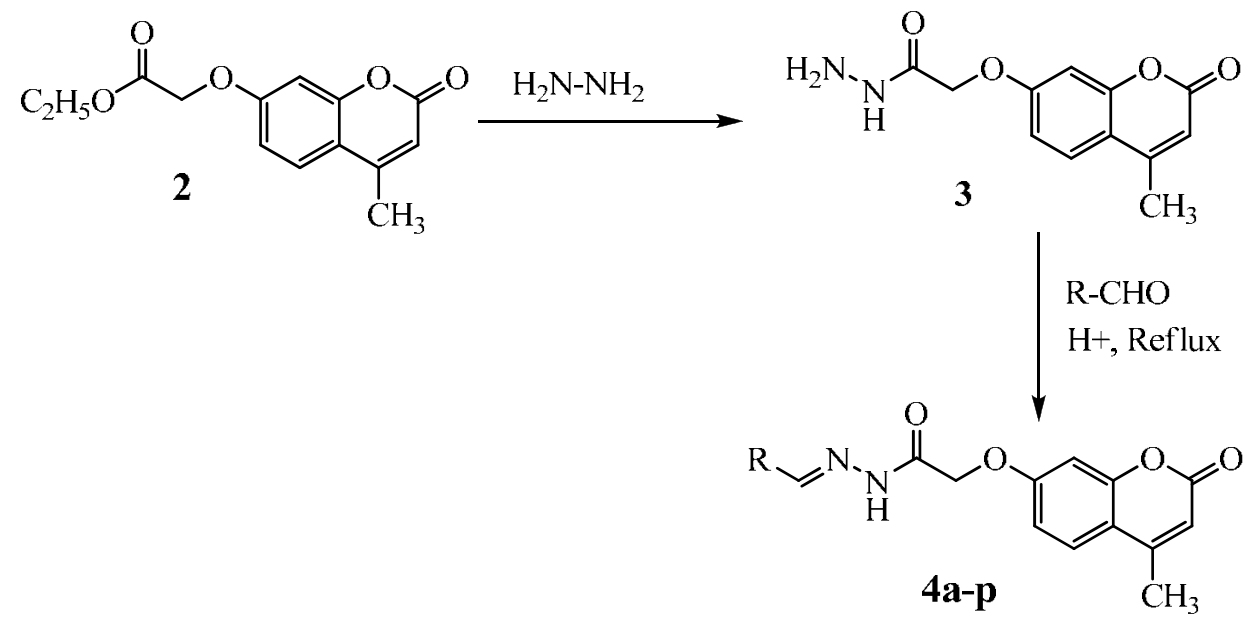

\section{Scheme 2}

The synthesis of $4 \mathbf{a}$ was optimized by varying the solvents, reaction time, temperature and the results are given in Table 1. It was found that the yield was up to $83.4 \%$ when the reaction mixture was refluxed for $30 \mathrm{~min}$. in a chloroform/methanol mixture.

Table 1. Yield of $4 \mathbf{a}$ at different reaction conditions

\begin{tabular}{ccccc}
\hline Entry & Solvent & Time $(\mathrm{min})$ & Temp. $\left({ }^{\circ} \mathrm{C}\right)$ & Yield $(\%)$ \\
\hline 1 & Ethanol & 30 & Reflux & 65.6 \\
2 & Ethanol & 60 & Reflux & 77.5 \\
3 & $\mathrm{CHCl}_{3}+$ Methanol & 20 & Reflux & 82.6 \\
4 & $\mathrm{CHCl}_{3}+$ Methanol & 30 & Reflux & 83.4 \\
5 & $\mathrm{CHCl}_{3}+$ Methanol & 60 & Reflux & 86.2 \\
6 & $\mathrm{CHCl}_{3}+$ Methanol & 30 & $75-80$ & 81.1 \\
\hline
\end{tabular}

In microwave assisted synthesis, the mixture of $\mathbf{3}$ with various aryl/hetero aromatic aldehydes is irradiated in an unmodified domestic microwave oven (800W) at $10 \%$ intensity for 2-3 min. to yield the range of desired products 4a-p depicted in Table 2. Optimal reaction time for the synthesis was 2-3 min. at $80 \mathrm{~W}$ of microwave irradiation power. Similarly the synthesis of compounds $\mathbf{2}$ and $\mathbf{3}$ was also attempted under the microwave conditions and detailed procedures are included in the experimental part. The study clearly showed that all the steps involved in this synthesis resulted in higher yields under micro wave conditions than the conventional method. 
Table 2. Comparison between microwave-assisted and conventional method of synthesis of 4a-p in terms of yield and time

\begin{tabular}{|c|c|c|c|c|c|c|}
\hline \multirow[b]{2}{*}{ Entry } & \multirow[b]{2}{*}{$\mathrm{R}$} & \multicolumn{2}{|c|}{ Microwave } & \multicolumn{2}{|c|}{ Conventional } & \multirow[b]{2}{*}{ M.p. $\left({ }^{\circ} \mathrm{C}\right)$} \\
\hline & & $\begin{array}{l}\text { Time } \\
(\mathrm{min})\end{array}$ & $\begin{array}{l}\text { Yield } \\
(\%)\end{array}$ & $\begin{array}{l}\text { Time } \\
(\mathrm{min})\end{array}$ & $\begin{array}{l}\text { Yield } \\
(\%)\end{array}$ & \\
\hline $4 a$ & & 2 & 92.3 & 60 & 86.2 & $276-278$ \\
\hline $4 b$ & & 2 & 91.7 & 60 & 83.5 & $296-298$ \\
\hline $4 c$ & & 2.5 & 86.4 & 90 & 81.6 & $266-268$ \\
\hline $4 d$ & & 2.5 & 87.6 & 60 & 84.3 & $280-282$ \\
\hline $4 e$ & & 3 & 80.5 & 120 & 76.8 & $258-260$ \\
\hline $4 f$ & & 3 & 80.1 & 120 & 69.7 & $260-262$ \\
\hline $4 g$ & & 3 & 88.2 & 120 & 77.5 & $252-254$ \\
\hline $4 h$ & & 2.5 & 93.5 & 60 & 83.2 & $266-268$ \\
\hline
\end{tabular}


Table 2. Continued

\begin{tabular}{|c|c|c|c|c|c|c|}
\hline \multirow[b]{2}{*}{ Entry } & \multirow[b]{2}{*}{$\mathrm{R}$} & \multicolumn{2}{|c|}{ Microwave } & \multicolumn{2}{|c|}{ Conventional } & \multirow[b]{2}{*}{ M.p. $\left({ }^{\circ} \mathrm{C}\right)$} \\
\hline & & $\begin{array}{l}\text { Time } \\
(\mathrm{min})\end{array}$ & $\begin{array}{l}\text { Yield } \\
(\%)\end{array}$ & $\begin{array}{l}\text { Time } \\
(\mathrm{min})\end{array}$ & $\begin{array}{l}\text { Yield } \\
(\%)\end{array}$ & \\
\hline $4 i$ & & 3 & 87.8 & 120 & 79.3 & $220-222$ \\
\hline $4 \mathbf{j}$ & & 2 & 93.6 & 60 & 89.4 & $288-290$ \\
\hline $4 k$ & & 2.5 & 86.2 & 120 & 69.6 & $260-262$ \\
\hline 41 & & 2.5 & 83.5 & 120 & 64.8 & $284-286$ \\
\hline $4 m$ & & 2.5 & 84.7 & 120 & 66.1 & $272-274$ \\
\hline $4 n$ & & 2 & 92.7 & 60 & 86.1 & $274-276$ \\
\hline 40 & & 3 & 74.2 & 150 & 61.2 & $256-258$ \\
\hline $4 p$ & & 2.5 & 81.3 & 120 & 72.6 & $270-272$ \\
\hline
\end{tabular}




\section{Antimicrobial activity}

Compounds 4c-l were screened for their antibacterial activity against gram-positive Staphylococcus aureus, Bacillus subtilis and gram-negative Escherichia coli, Pseudomonas aeruginase, Klebsiella pseumoniae by the disc diffusion method in Mueller-Hinton agar medium at three concentrations $(150,200,1000 \mathrm{ppm})$ in DMSO. These solutions were added to each filter disc, and the plates were incubated at $37{ }^{\circ} \mathrm{C}$ and examined for zone of inhibition around each disc after 48 hours. Results were compared with the activity of the standard antibiotic Ciproflaxacin. The results are summarized in Table 3.

According to preliminary antibacterial screening by the paper disc method, compounds $\mathbf{4 c}$, $\mathbf{4 e}, \mathbf{4 g}, \mathbf{4 h}, \mathbf{4 j}$ and $\mathbf{4 l}$ were found to be active against all the bacterial strains. The antibacterial activity of the synthesized compounds against standard and pathological strains was found to be similar (statistically insignificant difference). Most of the compounds exhibited mild to moderate antibacterial activity. All the compounds $4 \mathbf{c}-\mathbf{l}$ were effective against Pseudomonas aeruginosa ATCC 2853 (1000 ppm), Staphylococcus aureus ATCC 25923 (1000 ppm), Escherichia coli ATCC 25922 (1000 ppm) and Klebsiella pneumonia ATCC 13883 (1000 ppm). Compounds 4k-l, $\mathbf{4 j}, \mathbf{4 c}, \mathbf{4 h}$ and $\mathbf{4 e}$ exhibited highest activity against Pseudomonas aeruginosa ATCC 2853 (1000 ppm), Staphylococcus aureus ATCC 25923 (150 ppm), Bacillus subtilis ATCC 29212 (150 ppm), Klebsiella pneumoniae ATCC 13883 (150 ppm) and Bacillus subtilis ATCC 29212 (150 ppm) respectively. Compounds $\mathbf{4 d}, \mathbf{4 g}, \mathbf{4 i}$ and $\mathbf{4 f}$ exhibited highest activity against Escherichia coli ATCC 25922 (1000 ppm) and Pseudomonas aeruginosa ATCC 2853 (1000 ppm) respectively.

Compounds $\mathbf{4 k}, \mathbf{4 l}$ and $\mathbf{4 e}$ were found to be the most active compounds against the screened gram positive and gram negative standard and pathological bacterial strains. Based on the above results, we can conclude that compounds $\mathbf{4 g}, \mathbf{4 l}$ and $\mathbf{4 k}$ are most effective as it inhibits $18 \mathrm{~mm}$, $15 \mathrm{~mm}$ and $16 \mathrm{~mm}$ diameter growth of Staphylococcus aureus, Pseudomonas auregenosa.

Table 3. Antibacterial activity of compounds $4 \mathbf{c}-\mathbf{I}$

\begin{tabular}{|c|c|c|c|c|c|c|c|c|c|c|}
\hline \multicolumn{11}{|c|}{ Antibacterial activity* } \\
\hline \multirow[t]{2}{*}{ Compound } & \multicolumn{3}{|c|}{ S.aureus } & \multicolumn{3}{|c|}{$\begin{array}{c}\text { Klebsiella } \\
\text { Pneumonia }\end{array}$} & \multicolumn{2}{|c|}{$\begin{array}{l}\text { Bacillus } \\
\text { subtilis }\end{array}$} & \multirow{2}{*}{$\begin{array}{l}\text { E.coli } \\
1000^{\mathrm{a}}\end{array}$} & \multirow{2}{*}{$\begin{array}{l}\text { P.aeruginosa } \\
1000^{\mathrm{a}}\end{array}$} \\
\hline & $1000^{\mathrm{a}}$ & $200^{\mathrm{a}}$ & $150^{\mathrm{a}}$ & $1000^{\mathrm{a}}$ & $200^{\mathrm{a}}$ & $150^{\mathrm{a}}$ & $200^{\mathrm{a}}$ & $150^{\mathrm{a}}$ & & \\
\hline $4 c$ & 11 & - & 7 & 9 & 10 & 8 & 10 & 11 & 10 & 8 \\
\hline $4 d$ & 10 & - & - & 9 & - & 6 & 7 & 9 & 11 & 9 \\
\hline $4 e$ & 9 & 9 & - & 11 & 10 & 9 & 9 & 11 & 7 & 10 \\
\hline $4 f$ & 9 & - & 6 & 8 & 10 & - & 7 & 8 & 9 & 11 \\
\hline
\end{tabular}


Table 3. Continued

\begin{tabular}{|c|c|c|c|c|c|c|c|c|c|c|}
\hline \multicolumn{11}{|c|}{ Antibacterial activity* } \\
\hline \multirow[t]{2}{*}{ Compound } & \multicolumn{3}{|c|}{ S.aureus } & \multicolumn{3}{|c|}{$\begin{array}{c}\text { Klebsiella } \\
\text { Pneumonia }\end{array}$} & \multicolumn{2}{|c|}{$\begin{array}{l}\text { Bacillus } \\
\text { subtilis }\end{array}$} & \multirow{2}{*}{$\begin{array}{l}\text { E.coli } \\
1000^{\mathrm{a}} \\
\end{array}$} & \multirow{2}{*}{$\begin{array}{l}\text { P.aeruginosa } \\
1000^{\mathrm{a}}\end{array}$} \\
\hline & $1000^{\mathrm{a}}$ & $200^{\mathrm{a}}$ & $150^{\mathrm{a}}$ & $1000^{\mathrm{a}}$ & $200^{\mathrm{a}}$ & $150^{\mathrm{a}}$ & $200^{\mathrm{a}}$ & $150^{\mathrm{a}}$ & & \\
\hline $4 g$ & 18 & 5 & 6 & 10 & 10 & 9 & 6 & 6 & 12 & 7 \\
\hline $4 h$ & 9 & - & - & 7 & 8 & 10 & 8 & 9 & 10 & 11 \\
\hline $4 \mathbf{i}$ & 10 & - & - & 10 & 11 & 7 & 8 & 7 & 9 & 8 \\
\hline $4 \mathbf{j}$ & 13 & 7 & 9 & 10 & 9 & 10 & - & 10 & 6 & 11 \\
\hline $4 k$ & 11 & - & 8 & 8 & 11 & - & - & 10 & 10 & 16 \\
\hline 41 & 10 & 8 & - & 11 & 5 & 10 & 7 & 5 & 8 & 15 \\
\hline Ciprofloxacin ${ }^{b}$ & 26 & 15 & 13 & 20 & 25 & 15 & 16 & 16 & 16 & 18 \\
\hline
\end{tabular}

"Zone of inhibiton in mm.

${ }^{\mathrm{a}}$ In DMSO, Concentration in ppm. ${ }^{\mathrm{b}}$ Standard reference.

\section{Conclusions}

To conclude, the modified procedure for the conversion of $\mathbf{1}$ into $\mathbf{2}$ with dry DMF at $80{ }^{\circ} \mathrm{C}$ instead of dry acetone resulted with improved yield of $82 \%$. The same conversion also effected by sodium bismuthate as a catalyst instead of $\mathrm{K}_{2} \mathrm{CO}_{3}$ resulted only $40 \%$ yield. The new Schiff base 2-[(4-Methyl-2-oxo-2H-chromen-7-yl)oxy]-N'-(substitutedmethylene)acetohydrazides (4ap) have been synthesised by reaction of 2-[(4-Methyl-2-oxo-2H-chromen-7yl)oxy]acetohydrazide (3) with aryl/hetero aromatic aldehydes under conventional and microwave irradiation methods. The compounds $\mathbf{4 c - l}$ were tested for antibacterial acivity by disc diffusion method, showing moderate to potent inhibition.

\section{Experimental Section}

General Procedures. Infrared (IR) spectra were recorded at room temperature from $4000 \mathrm{~cm}^{-1}$ to $400 \mathrm{~cm}^{-1}$ with $\mathrm{KBr}$ pellets at a resolution of $4 \mathrm{~cm}^{-1}$, using Avatar 330 equipped with DTGS detector. Most of the obtained vibrational bands of the IR spectrum were identified and 
compared with those available in literature. The ${ }^{1} \mathrm{H}$ NMR was measured on a Bruker AMX-400 instrument at room temperature using the X-WIN NMR version X-WIN NMR $1.3 \mathrm{cn}$ drx software. The ${ }^{1} \mathrm{H}$ NMR was measured for $\sim 0.03 \mathrm{M}$ solutions in DMSO- $\mathrm{d}_{6}$ using TMS as internal reference. The accuracy of the ${ }^{1} \mathrm{H}$ shifts is considered to be $0.02 \mathrm{ppm}$. The coupling constants $\mathrm{J}$ are in Hz. Mass spectra were obtained using LC-MS-MS (3200 Q-trap). Melting points were determined in open capillaries and are uncorrected. All reagents were purchased from Aldrich and Qualigens and used without further purification.

\section{Procedure for the preparation of Ethyl 2-[(4-Methyl-2-oxo-2H-chromen-7-yl)oxy]acetate} (2)

(a) Conventional method using $\mathrm{K}_{2} \mathrm{CO}_{3}$. Method A. To a solution of 7-Hydroxy-4-methyl-2Hchromen-2-one 1 in dry DMF, anhydrous potassium carbonate (1.0 molar equiv) and ethyl chloro acetate $\left(1.0\right.$ molar equiv) were added. The resultant mixture was stirred at $80{ }^{\circ} \mathrm{C}$ for $10 \mathrm{~h}$, cooled and then the reaction mixture was added to a large amount of water. The solid separated was filtered, washed with excess of water. The crude product was purified by crystallization from ethanol. The yield of product was $81-82 \%$ [lit. yield $40 \%]^{24}$.

(b) Conventional method using $\mathrm{NaBiO}_{3}$. Method $\mathrm{B}$. To a solution of 7-Hydroxy-4-methyl-2Hchromen-2-one 1 in dry DMF, anhydrous sodium bismuthate (1.0 molar equiv) and ethyl chloro acetate $\left(1.0\right.$ molar equiv) were added. The resultant mixture was stirred at $80{ }^{\circ} \mathrm{C}$ for $14 \mathrm{~h}$, cooled, filtered the reaction mixture and then the filtrate was added to a large amount of water. The solid separated was filtered and then the filtrate was extracted with ethyl acetate. The organic layer was separated and evaporated the solvent. The crude product was purified by crystallization from ethanol. The yield of product was 41-42.5\% [lit. yield $40 \%]^{24}$.

(c) Solvent free microwave irradiation method. Method $\mathrm{C}$. This involves the irradiation of a mixture of 7-Hydroxy-4-methyl-2H-chromen-2-one 1 ( 0.01 mole), chloroethyl acetate $(0.01$ mole) and potassium carbonate ( 0.01 mole) in a microwave oven (MW domestic type oven $800 \mathrm{~W}$ SANYO) at $10 \%$ intensity for $12 \mathrm{~min}$. (six pulses each of $2 \mathrm{~min}$.). After completion of reaction (by TLC), the mixture was poured into ice-cold water. The separated solid was filtered, washed with excess of cold water and dried at room temperature. The yield of product was 4445.2 \%. M.p. $94-96{ }^{\circ} \mathrm{C}\left[\right.$ lit. $\left.88-90{ }^{\circ} \mathrm{C}\right] ;{ }^{24} \mathrm{IR}(\mathrm{KBr})$ v: $2999,2917,1765,1724,1615,1391,1196$, $1508,1476,1196,1080 \mathrm{~cm}^{-1} ;{ }^{1} \mathrm{H}$ NMR (400 MHz, DMSO, ppm), $\delta=7.69(1 \mathrm{H}, \mathrm{d}, \mathrm{J}=9.5 \mathrm{~Hz}, 6-$ $\mathrm{H}), 6.99(2 \mathrm{H}, \mathrm{m}, 5,8-\mathrm{H}), 6.23(1 \mathrm{H}, \mathrm{s}, 3-\mathrm{H}), 4.93\left(2 \mathrm{H}, \mathrm{s}, \mathrm{OCH}_{2}\right), 4.18\left(2 \mathrm{H}, \mathrm{q}, \mathrm{J}=7.0 \mathrm{~Hz}, \mathrm{CH}_{2}\right)$, $2.39\left(3 \mathrm{H}, \mathrm{s}, \mathrm{CH}_{3}\right), 1.22\left(3 \mathrm{H}, \mathrm{t}, \mathrm{J}=7.0 \mathrm{~Hz}, \mathrm{CH}_{3}\right) ; \mathrm{LC}-\mathrm{MS}: \mathrm{m} / \mathrm{z} 263.0(\mathrm{M}+1)$.

Procedure for the preparation of 2-[(4-Methyl-2-oxo- $2 \mathrm{H}$-chromen-7-yl)oxy]acetohydrazide (3). Ethyl 2-[(4-methyl-2-oxo-2H-chromen-7-yl)oxy]acetate (3, $0.01 \mathrm{~mole})$ in ethanol $(20 \mathrm{~mL})$ was stirred at room temperature for $20 \mathrm{~min}$. To this mixture hydrazine hydrate $(0.014 \mathrm{~mole})$ was added. The resultant mixture was stirred at room temperature for $15 \mathrm{~min}$, and the solid filtered with a sintered glass funnel. The residue was dried and then desiccated to afford a crystalline powder. The powder was recrystallised from chloroform/methanol and gave colorless needles. The yield of product was $88-90 \%$. M.p. $202-204{ }^{\circ} \mathrm{C}\left[\right.$ lit. $\left.198-200{ }^{\circ} \mathrm{C}\right] ;{ }^{24} \mathrm{IR}(\mathrm{KBr})$ v: 3331,3268 , 
3082, 2958, 1731, 1676, 1609, 1508, 1439, 1153, $1074 \mathrm{~cm}^{-1}$; ${ }^{1} \mathrm{H}$ NMR (400 MHz, DMSO, ppm), $\delta=9.41(1 \mathrm{H}, \mathrm{s}, \mathrm{NH}), 7.69(1 \mathrm{H}, \mathrm{d}, \mathrm{J}=8.8 \mathrm{~Hz}, 6-\mathrm{H}), 7.00(1 \mathrm{H}, \mathrm{dd}, \mathrm{J}=8.8 \mathrm{~Hz}, \mathrm{~J}=2.4 \mathrm{~Hz}, 5-\mathrm{H}), 6.9$ $(1 \mathrm{H}, \mathrm{d}, \mathrm{J}=10.3 \mathrm{~Hz}, 8-\mathrm{H}), 6.21(1 \mathrm{H}, \mathrm{s}, 3-\mathrm{H}), 4.61\left(2 \mathrm{H}, \mathrm{s}, \mathrm{NH}_{2}\right), 4.35\left(2 \mathrm{H}, \mathrm{s}, \mathrm{OCH}_{2}\right), 2.39(3 \mathrm{H}, \mathrm{s}$, $\left.\mathrm{CH}_{3}\right)$; LC-MS: m/z $249.0(\mathrm{M}+1)$.

\section{Procedure for the synthesis of 2-[(4-Methyl-2-oxo-2H-chromen-7-yl)oxy]-N'-(substituted methylene)acetohydrazides (4a-p)}

Conventional. A mixture of compound 3 (0.01 mole) in chloroform/methanol (1:1) mixture (30 $\mathrm{mL})$, aryl / hetero aromatic aldehyde $(0.01$ mole) and $1 \mathrm{~mL}$ of glacial acetic acid was refluxed on a water bath for $60-150$ minutes. The mixture was allowed to cool, and then the separated solid was filtered, washed with excess of methanol.

Microwave. This involves the irradiation of a mixture of compound 3 (0.01 mole) and aryl / heterocyclic aldehyde $(0.01$ mole) in a microwave oven (MW domestic type oven $800 \mathrm{~W}$ SANYO) at $10 \%$ intensity for 2-3 min. (four to six pulses each of $30 \mathrm{sec}$.) and the product was set aside to cool. The separated solid was filtered, washed with excess of methanol and dried. When solid aldehyde was used, the mixture of compound 3 ( 0.01 mole), aryl / hetero aromatic aldehyde $(0.01 \mathrm{~mole})$ and alumina $\left(1.0 \mathrm{~g}\right.$, mesh: $\left.70-290 \mathrm{ASTM},{ }_{\mathrm{pH}}=4.5\right)$ were finely ground in mortar and pestle then, the mixture was irradiated in microwave oven (MW domestic type oven $800 \mathrm{~W}, \mathrm{SANYO}$ ) at $10 \%$ intensity for $2-3 \mathrm{~min}$. (four to six pulses each of $30 \mathrm{sec}$.) and set aside to cool. The resultant solid mixture was poured into $30 \mathrm{~mL}$ of methanol, and then solid separated was filtered, washed with methanol.

2-[(4-Methyl-2-oxo-2H-chromen-7-yl)oxy]- $N$ '-(benzylidene)acetohydrazide (4a). M.p. 276$278{ }^{\circ} \mathrm{C}$; IR (KBr) v: 3072, 2969, 1712, 1686, 1615, 1511, 1435, 1393, 1271, 1160, $1084 \mathrm{~cm}^{-1} ;{ }^{1} \mathrm{H}$ NMR (400 MHz, DMSO, ppm), $\delta=11.66(1 \mathrm{H}, \mathrm{d}, \mathrm{J}=7.5 \mathrm{~Hz}, \mathrm{HC}=\mathrm{N}), 8.32 \& 8.02(1 \mathrm{H}, 2 \mathrm{~s}, \mathrm{NH})$, $7.72(1 \mathrm{H}, \mathrm{s}, 6-\mathrm{H}), 7.43(5 \mathrm{H}, \mathrm{s}, \mathrm{Ph}-\mathrm{H}), 7.04(2 \mathrm{H}, \mathrm{d}, \mathrm{J}=18.7 \mathrm{~Hz}, 5,8-\mathrm{H}), 6.23(1 \mathrm{H}, \mathrm{d}, \mathrm{J}=8.2 \mathrm{~Hz}, 3-$ $\mathrm{H}), 5.30\left(1 \mathrm{H}, \mathrm{s}, \mathrm{OCH}_{2}\right), 4.81\left(1 \mathrm{H}, \mathrm{s}, \mathrm{OCH}_{2}\right), 2.40\left(3 \mathrm{H}, \mathrm{s}, \mathrm{CH}_{3}\right) ; \mathrm{LC}-\mathrm{MS}: \mathrm{m} / \mathrm{z} 335(\mathrm{M}-1)$.

2-[(4-Methyl-2-oxo-2H-chromen-7-yl)oxy]- $\boldsymbol{N}^{\prime}$-(4-chlorobenzylidene)acetohydrazide $\quad \mathbf{( 4 b )}$. M.p. 296-298 ${ }^{\circ} \mathrm{C}$; IR (KBr) v: 3188, 3065, 2966, 1711, 1687, 1617, 1511, 1435, 1390, 1270, $1139,1084 \mathrm{~cm}^{-1} ;{ }^{1} \mathrm{H}$ NMR (400 MHz, DMSO, ppm), $\delta=11.70(1 \mathrm{H}, \mathrm{s}, \mathrm{HC}=\mathrm{N}), 8.32 \& 8.01(1 \mathrm{H}$, $2 \mathrm{~s}, \mathrm{NH}), 7.76\left(2 \mathrm{H}, \mathrm{d}, \mathrm{J}=8.3 \mathrm{~Hz}, 3^{\prime}, 5^{\prime}-\mathrm{H}\right), 7.71(1 \mathrm{H}, \mathrm{dd}, \mathrm{J}=8.6 \mathrm{~Hz}, 6-\mathrm{H}), 7.51(2 \mathrm{H}, \mathrm{d}, \mathrm{J}=8.3 \mathrm{~Hz}$, $\left.2^{\prime}, 6^{\prime}-\mathrm{H}\right), 7.00(2 \mathrm{H}, \mathrm{d}, \mathrm{J}=11.9 \mathrm{~Hz}, 5,8-\mathrm{H}), 6.23(1 \mathrm{H}, \mathrm{d}, \mathrm{J}=8.2 \mathrm{~Hz}, 3-\mathrm{H}), 5.30\left(1 \mathrm{H}, \mathrm{s}, \mathrm{OCH}_{2}\right), 4.81$ $\left(1 \mathrm{H}, \mathrm{s}, \mathrm{OCH}_{2}\right), 2.40\left(3 \mathrm{H}, \mathrm{s}, \mathrm{CH}_{3}\right)$; LC-MS: m/z $371(\mathrm{M}+1)$.

2-[(4-Methyl-2-oxo-2H-chromen-7-yl)oxy]- $N$ '-(3-nitrobenzylidene)acetohydrazide (4c). M.p. 266-268 ${ }^{\circ} \mathrm{C}$; IR (KBr) v: 3073, 2958, 1720, 1687, 1617, 1529, 1433, 1387, 1347, 1275, 1138 , $1085 \mathrm{~cm}^{-1} ;{ }^{1} \mathrm{H}$ NMR $(400 \mathrm{MHz}, \mathrm{DMSO}, \mathrm{ppm}), \delta=11.88(1 \mathrm{H}, \mathrm{s}, \mathrm{HC}=\mathrm{N}), 8.52 \& 8.45(1 \mathrm{H}, 2 \mathrm{~s}$, $\mathrm{NH}), 8.25\left(1 \mathrm{H}, \mathrm{d}, \mathrm{J}=5.8 \mathrm{~Hz}, 6^{\prime}-\mathrm{H}\right), 8.21\left(1 \mathrm{H}, \mathrm{d}, \mathrm{J}=7.7 \mathrm{~Hz}, 4^{\prime}-\mathrm{H}\right), 8.15\left(1 \mathrm{H}, \mathrm{s}, 2^{\prime}-\mathrm{H}\right), 7.8(1 \mathrm{H}, \mathrm{t}, \mathrm{J}$ $\left.=5.5 \mathrm{~Hz}, 5^{\prime}-\mathrm{H}\right), 7.71(1 \mathrm{H}, \mathrm{d}, \mathrm{J}=9.0 \mathrm{~Hz}, 6-\mathrm{H}), 7.01(2 \mathrm{H}, \mathrm{d}, \mathrm{J}=10.3 \mathrm{~Hz}, 5,8-\mathrm{H}), 5.36(1 \mathrm{H}, \mathrm{s}$, $\left.\mathrm{OCH}_{2}\right), 4.85\left(1 \mathrm{H}, \mathrm{s}, \mathrm{OCH}_{2}\right), 2.40\left(3 \mathrm{H}, \mathrm{s}, \mathrm{CH}_{3}\right) ; \mathrm{LC}-\mathrm{MS}: \mathrm{m} / \mathrm{z} 382(\mathrm{M}+1)$.

2-[(4-Methyl-2-oxo-2H-chromen-7-yl)oxy]- $N$ '-(4-hydroxybenzylidene)acetohydrazide (4d). M.p. $280-282{ }^{\circ} \mathrm{C}$; IR (KBr) v: 3280,3079, 2908, 1717, 1670, 1610, 1512, 1423, 1390, 1356, 
1260, 1145, $1086 \mathrm{~cm}^{-1}$; ${ }^{1} \mathrm{H}$ NMR (400 MHz, DMSO, ppm), $\delta=11.43(1 \mathrm{H}, \mathrm{d}, \mathrm{J}=15.9 \mathrm{~Hz}$, $\mathrm{HC}=\mathrm{N}), 9.90(1 \mathrm{H}, \mathrm{s}, \mathrm{OH}), 8.21 \& 7.91(1 \mathrm{H}, 2 \mathrm{~s}, \mathrm{NH}), 7.71(1 \mathrm{H}, \mathrm{d}, \mathrm{J}=8.7 \mathrm{~Hz}, 6-\mathrm{H}), 7.63(2 \mathrm{H}, \mathrm{d}, \mathrm{J}$ $\left.=8.4 \mathrm{~Hz}, 3^{\prime}, 5^{\prime}-\mathrm{H}\right), 7.00(2 \mathrm{H}, \mathrm{m}, 5,8-\mathrm{H}), 6.81\left(2 \mathrm{H}, \mathrm{d}, \mathrm{J}=8.4 \mathrm{~Hz}, 2^{\prime}, 6^{\prime}-\mathrm{H}\right), 6.22(1 \mathrm{H}, \mathrm{d}, \mathrm{J}=7.7 \mathrm{~Hz}$, 3-H), $5.25\left(1 \mathrm{H}, \mathrm{s}, \mathrm{OCH}_{2}\right), 4.77\left(1 \mathrm{H}, \mathrm{s}, \mathrm{OCH}_{2}\right), 2.40\left(3 \mathrm{H}, \mathrm{s}, \mathrm{CH}_{3}\right)$; LC-MS: m/z $353(\mathrm{M}+1)$.

2-[(4-Methyl-2-oxo-2H-chromen-7-yl)oxy]- $\boldsymbol{N}^{\prime}$-(4-hydroxy-3-methoxybenzylidene)aceto hydrazide (4e). M.p. 258-260 ${ }^{\circ} \mathrm{C}$; IR (KBr) v: 3482, 3135, 3086, 2975, 1710, 1697, 1612, 1518 , 1428, 1390, 1267, 1161, $1077 \mathrm{~cm}^{-1}$; ${ }^{1} \mathrm{H}$ NMR (400 MHz, DMSO, ppm), $\delta=11.46(1 \mathrm{H}, \mathrm{d}, \mathrm{J}=$ $23.8 \mathrm{~Hz}, \mathrm{HC}=\mathrm{N}), 9.50(1 \mathrm{H}, \mathrm{s}, \mathrm{OH}), 8.19 \& 7.90(1 \mathrm{H}, 2 \mathrm{~s}, \mathrm{NH}), 7.71(1 \mathrm{H}, \mathrm{d}, \mathrm{J}=7.8 \mathrm{~Hz}, 6-\mathrm{H}), 7.27$ $(1 \mathrm{H}, \mathrm{d}, \mathrm{J}=9.9 \mathrm{~Hz}, 8-\mathrm{H}), 6.98\left(3 \mathrm{H}, \mathrm{m}, 2^{\prime}, 5^{\prime}, 6^{\prime}-\mathrm{H}\right), 6.82(1 \mathrm{H}, \mathrm{dd}, \mathrm{J}=2.8 \mathrm{~Hz}, \mathrm{~J}=8.1 \mathrm{~Hz}, 5-\mathrm{H}), 6.22$ $(1 \mathrm{H}, \mathrm{d}, \mathrm{J}=8.8 \mathrm{~Hz}, 3-\mathrm{H}), 5.28\left(1 \mathrm{H}, \mathrm{s}, \mathrm{OCH}_{2}\right), 4.78\left(1 \mathrm{H}, \mathrm{s}, \mathrm{OCH}_{2}\right), 3.80\left(3 \mathrm{H}, \mathrm{s}, \mathrm{OCH}_{3}\right), 2.40(3 \mathrm{H}, \mathrm{s}$, $\left.\mathrm{CH}_{3}\right)$; LC-MS: m/z $383(\mathrm{M}+1)$.

2-[(4-Methyl-2-oxo-2H-chromen-7-yl)oxy]- $\boldsymbol{N}^{\prime}$-(4-dimethylaminobenzylidene)aceto hydrazide (4f). M.p. $260-262{ }^{\circ} \mathrm{C}$; IR (KBr) v: 3316, 2913, 1706, 1683, 1615, 1525, 1444, 1366, 1270, 1152, $1081 \mathrm{~cm}^{-1}$; ${ }^{1} \mathrm{H}$ NMR (400 MHz, DMSO, ppm), $\delta=11.36(1 \mathrm{H}, \mathrm{d}, \mathrm{J}=20.9 \mathrm{~Hz}$, $\mathrm{HC}=\mathrm{N}), 8.16 \& 8.31(1 \mathrm{H}, 2 \mathrm{~s}, \mathrm{NH}), 7.71(1 \mathrm{H}, \mathrm{d}, \mathrm{J}=4.8 \mathrm{~Hz}, 6-\mathrm{H}), 7.51\left(2 \mathrm{H}, \mathrm{d}, \mathrm{J}=5.3 \mathrm{~Hz}, 3^{\prime}, 5^{\prime}-\right.$ $\mathrm{H}), 7.0(2 \mathrm{H}, \mathrm{m}, 5,8-\mathrm{H}), 6.73\left(2 \mathrm{H}, \mathrm{d}, \mathrm{J}=5.3 \mathrm{~Hz}, 2^{\prime}, 6^{\prime}-\mathrm{H}\right), 6.22(1 \mathrm{H}, \mathrm{d}, \mathrm{J}=8.2 \mathrm{~Hz}, 3-\mathrm{H}), 5.24(1 \mathrm{H}$, s, $\left.\mathrm{OCH}_{2}\right), 4.76\left(1 \mathrm{H}, \mathrm{s}, \mathrm{OCH}_{2}\right), 2.96\left(6 \mathrm{H}, \mathrm{s}, \mathrm{N}\left(\mathrm{CH}_{3}\right)_{2}\right), 2.40\left(3 \mathrm{H}, \mathrm{s}, \mathrm{CH}_{3}\right) ; \mathrm{LC}-\mathrm{MS}: \mathrm{m} / \mathrm{z} 378(\mathrm{M}-1)$.

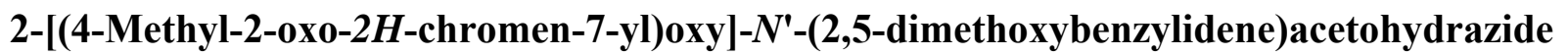
(4g). M.p. 252-254 ${ }^{\circ} \mathrm{C}$; IR (KBr) v: 3271, 3094, 2979, 1715, 1695, 1621, 1535, 1494, 1361, 1297, 1150, $1080 \mathrm{~cm}^{-1}$; ${ }^{1} \mathrm{H}$ NMR (400 MHz, DMSO, ppm), $\delta=11.65(1 \mathrm{H}, \mathrm{d}, \mathrm{J}=16.2 \mathrm{~Hz}$, $\mathrm{HC}=\mathrm{N}), 8.31 \& 8.64(1 \mathrm{H}, 2 \mathrm{~s}, \mathrm{NH}), 7.71(1 \mathrm{H}, \mathrm{d}, \mathrm{J}=6.2 \mathrm{~Hz}, 6-\mathrm{H}), 7.35\left(1 \mathrm{H}, 2 \mathrm{~d}, \mathrm{~J}=3.8 \mathrm{~Hz}, 6^{\prime}-\mathrm{H}\right)$, $7.01\left(4 \mathrm{H}, \mathrm{m}, 5,8,3^{\prime}, 4^{\prime}-\mathrm{H}\right), 6.22(1 \mathrm{H}, \mathrm{d}, \mathrm{J}=8.5 \mathrm{~Hz}, 3-\mathrm{H}), 5.30\left(1 \mathrm{H}, \mathrm{s}, \mathrm{OCH}_{2}\right), 4.78(1 \mathrm{H}, \mathrm{s}$, $\left.\mathrm{OCH}_{2}\right), 3.80\left(3 \mathrm{H}, \mathrm{s}, 2^{\prime}-\mathrm{H}\right), 3.73\left(3 \mathrm{H}, \mathrm{s}, 5^{\prime}-\mathrm{H}\right), 2.40\left(3 \mathrm{H}, \mathrm{s}, \mathrm{CH}_{3}\right)$; LC-MS: m/z 395 (M-1).

2-[(4-Methyl-2-oxo-2H-chromen-7-yl)oxy]- $N^{\prime}$-(3-chlorobenzylidene)acetohydrazide (4h). M.p. 266-268 ${ }^{\circ} \mathrm{C}$; IR (KBr) v: 3101, 2976, 1713, 1682, 1617, 1510, 1433, 1390, 1273, 1157 , $1083 \mathrm{~cm}^{-1} ;{ }^{1} \mathrm{H}$ NMR (400 MHz, DMSO, ppm), $\delta=11.76(1 \mathrm{H}, \mathrm{s}, \mathrm{HC}=\mathrm{N}), 8.30 \& 8.0(1 \mathrm{H}, 2 \mathrm{~s}$, $\mathrm{NH}), 7.75\left(1 \mathrm{H}, \mathrm{t}, 5^{\prime}-\mathrm{H}\right), 7.71(1 \mathrm{H}, \mathrm{d}, \mathrm{J}=8.7 \mathrm{~Hz}, 6-\mathrm{H}), 7.5\left(2 \mathrm{H}, \mathrm{t}, \mathrm{J}=4.9 \mathrm{~Hz}, 4^{\prime}, 6^{\prime}-\mathrm{H}\right), 7.02(2 \mathrm{H}$, m, 5, 8-H), $6.23(1 \mathrm{H}, \mathrm{d}, \mathrm{J}=8.9 \mathrm{~Hz}, 3-\mathrm{H}), 5.32\left(1 \mathrm{H}, \mathrm{s}, \mathrm{OCH}_{2}\right), 4.82\left(1 \mathrm{H}, \mathrm{s}, \mathrm{OCH}_{2}\right), 2.40(3 \mathrm{H}, \mathrm{s}$, $\left.\mathrm{CH}_{3}\right)$; LC-MS: m/z $371(\mathrm{M}+1)$.

2-[(4-Methyl-2-oxo-2H-chromen-7-yl)oxy]- $N^{\prime}$-[3,4-dimethoxybenzylidene]acetohydrazide (4i). M.p. 220-222 ${ }^{\circ} \mathrm{C}$; IR (KBr) v: 3189, 3086, 2927, 2834, 1731, 1682, 1615, 1509, 1421, 1391, 1267, 1155, $1080 \mathrm{~cm}^{-1}$; ${ }^{1} \mathrm{H}$ NMR (400 MHz, DMSO, ppm), $\delta=11.52(1 \mathrm{H}, \mathrm{d}, \mathrm{J}=23.5 \mathrm{~Hz}$, $\mathrm{HC}=\mathrm{N}), 8.24 \& 7.94(1 \mathrm{H}, 2 \mathrm{~s}, \mathrm{NH}), 7.70(1 \mathrm{H}, \mathrm{d}, \mathrm{J}=8.7 \mathrm{~Hz}, 6-\mathrm{H}), 7.31\left(1 \mathrm{H}, \mathrm{d}, \mathrm{J}=17.0 \mathrm{~Hz}, 5^{\prime}-\mathrm{H}\right)$, $7.20\left(1 \mathrm{H}, \mathrm{t}, \mathrm{J}=8.2 \mathrm{~Hz}, 6^{\prime}-\mathrm{H}\right), 7.01(2 \mathrm{H}, \mathrm{d}, \mathrm{J}=7.9 \mathrm{~Hz}, 5,8-\mathrm{H}), 6.96\left(1 \mathrm{H}, \mathrm{dd}, \mathrm{J}=2.4 \mathrm{~Hz}, 2^{\prime}-\mathrm{H}\right), 6.22$ $(1 \mathrm{H}, \mathrm{d}, \mathrm{J}=9.2 \mathrm{~Hz}, 3-\mathrm{H}), 5.30\left(1 \mathrm{H}, \mathrm{s}, \mathrm{OCH}_{2}\right), 4.79\left(1 \mathrm{H}, \mathrm{s}, \mathrm{OCH}_{2}\right), 3.81\left(6 \mathrm{H}, \mathrm{s},\left(\mathrm{OCH}_{3}\right)_{2}\right), 2.40$ $\left(3 \mathrm{H}, \mathrm{s}, \mathrm{CH}_{3}\right)$; LC-MS: m/z $397(\mathrm{M}+1)$.

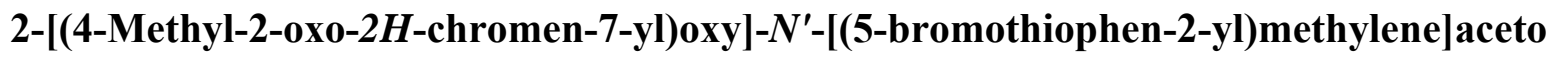
hydrazide (4j). M.p. 288-290 ${ }^{\circ} \mathrm{C}$; IR (KBr) v: 3053, 2917, 1713,1681, 1616, 1510, 1429, 1389 , 1274, 1139, $1083 \mathrm{~cm}^{-1}$; ${ }^{1} \mathrm{H}$ NMR (400 MHz, DMSO, ppm), $\delta=11.68(1 \mathrm{H}, \mathrm{d}, \mathrm{J}=21.8 \mathrm{~Hz}$, $\mathrm{HC}=\mathrm{N}), 8.47 \& 8.10(1 \mathrm{H}, 2 \mathrm{~s}, \mathrm{NH}), 7.71(1 \mathrm{H}, \mathrm{d}, \mathrm{J}=8.7 \mathrm{~Hz}, 6-\mathrm{H}), 7.28\left(2 \mathrm{H}, \mathrm{m}, 3^{\prime}, 4^{\prime}-\mathrm{H}\right), 6.99$ 
$(2 \mathrm{H}, \mathrm{m}, 5,8-\mathrm{H}), 6.23(1 \mathrm{H}, \mathrm{d}, \mathrm{J}=8.1 \mathrm{~Hz}, 3-\mathrm{H}), 5.18\left(1 \mathrm{H}, \mathrm{s}, \mathrm{OCH}_{2}\right), 4.78\left(1 \mathrm{H}, \mathrm{s}, \mathrm{OCH}_{2}\right), 2.40(3 \mathrm{H}$, s, $\left.\mathrm{CH}_{3}\right)$; LC-MS: m/z 320 (M-1), 322(M+1).

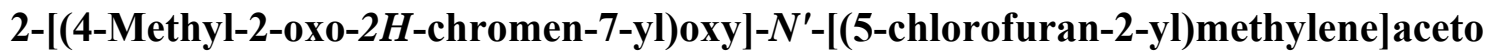
hydrazide (4k). M.p. 260-262 ${ }^{\circ} \mathrm{C}$; IR (KBr) v: 3052, 2928, 1715, 1686, 1616, 1512, 1434, 1393, 1272, 1159, $1084 \mathrm{~cm}^{-1} ;{ }^{1} \mathrm{H}$ NMR (400 MHz, DMSO, ppm), $\delta=11.68(1 \mathrm{H}, \mathrm{d}, \mathrm{J}=3.2 \mathrm{~Hz}, \mathrm{HC}=\mathrm{N})$, $8.14 \& 8.31(1 \mathrm{H}, 2 \mathrm{~s}, \mathrm{NH}), 7.71(1 \mathrm{H}, \mathrm{d}, \mathrm{J}=7.8 \mathrm{~Hz}, 6-\mathrm{H}), 6.99\left(1 \mathrm{H}, \mathrm{d}, \mathrm{J}=3.3 \mathrm{~Hz}, 4^{\prime}-\mathrm{H}\right), 6.96(1 \mathrm{H}$, $\left.\mathrm{d}, \mathrm{J}=3.3 \mathrm{~Hz}, 3^{\prime}-\mathrm{H}\right), 6.93(1 \mathrm{H}, \mathrm{d}, \mathrm{J}=3.2 \mathrm{~Hz}, 8-\mathrm{H}), 6.67(1 \mathrm{H}, \mathrm{d}, \mathrm{J}=4.6 \mathrm{~Hz}, 5-\mathrm{H}), 6.23(1 \mathrm{H}, \mathrm{d}, \mathrm{J}=$ $8.9 \mathrm{~Hz}, 3-\mathrm{H}), 5.22\left(1 \mathrm{H}, \mathrm{s}, \mathrm{OCH}_{2}\right), 4.80\left(1 \mathrm{H}, \mathrm{s}, \mathrm{OCH}_{2}\right), 2.40\left(3 \mathrm{H}, \mathrm{s}, \mathrm{CH}_{3}\right) ; \mathrm{LC}-\mathrm{MS}: \mathrm{m} / \mathrm{z} 358.9(\mathrm{M}-$ $1)$.

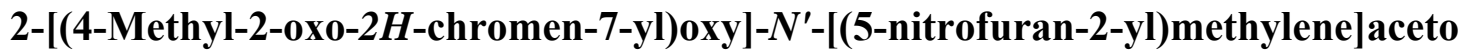
hydrazide (4I). M.p. $284-286^{\circ} \mathrm{C}$; IR (KBr) v: 3112, 1715, 1701, 1679, 1614, 1500, 1456, 1390, 1351, 1260, 1137, $1081 \mathrm{~cm}^{-1}$; ${ }^{1} \mathrm{H}$ NMR (400 MHz, DMSO, ppm), $\delta=12.03(1 \mathrm{H}, \mathrm{d}, \mathrm{J}=10.0 \mathrm{~Hz}$, $\mathrm{HC}=\mathrm{N}), 8.30 \& 7.97(1 \mathrm{H}, 2 \mathrm{~s}, \mathrm{NH}), 7.79\left(1 \mathrm{H}, \mathrm{dd}, \mathrm{J}=3.8 \mathrm{~Hz}, \mathrm{~J}=10.1 \mathrm{~Hz}, 4^{\prime}-\mathrm{H}\right), 7.71(1 \mathrm{H}, \mathrm{d}, \mathrm{J}=$ $8.6 \mathrm{~Hz}, 6-\mathrm{H}), 7.28\left(1 \mathrm{H}, \mathrm{dd}, \mathrm{J}=3.8 \mathrm{~Hz}, \mathrm{~J}=18.9 \mathrm{~Hz}, 3^{\prime}-\mathrm{H}\right), 7.00(2 \mathrm{H}, \mathrm{m}, 5,8-\mathrm{H}), 6.23(1 \mathrm{H}, \mathrm{d}, \mathrm{J}=$ $8.8 \mathrm{~Hz}, 3-\mathrm{H}), 5.28\left(1 \mathrm{H}, \mathrm{s}, \mathrm{OCH}_{2}\right), 4.86\left(1 \mathrm{H}, \mathrm{s}, \mathrm{OCH}_{2}\right), 2.40\left(3 \mathrm{H}, \mathrm{s}, \mathrm{CH}_{3}\right) ; \mathrm{LC}-\mathrm{MS}: \mathrm{m} / \mathrm{z} 372$ $(\mathrm{M}+1)$.

2-[(4-Methyl-2-oxo-2H-chromen-7-yl)oxy]- $N^{\prime}-[($ furan-3-yl)methylene]acetohydrazide (4m). M.p. $272-274{ }^{\circ} \mathrm{C}$; IR (KBr) v: 3110, 2958, 1710, 1688, 1617, 1511, 1433, 1390, 1272, 1158 , $1086 \mathrm{~cm}^{-1} ;{ }^{1} \mathrm{H}$ NMR (400 MHz, DMSO, ppm), $\delta=11.52(1 \mathrm{H}, \mathrm{d}, \mathrm{J}=21.7 \mathrm{~Hz}, \mathrm{HC}=\mathrm{N}), 8.28 \&$ $8.13(1 \mathrm{H}, 2 \mathrm{~s}, \mathrm{NH}), 7.97\left(1 \mathrm{H}, \mathrm{s}, 2^{\prime}-\mathrm{H}\right), 7.74\left(1 \mathrm{H}, \mathrm{s}, 5^{\prime}-\mathrm{H}\right), 7.71(1 \mathrm{H}, \mathrm{d}, \mathrm{J}=8.7 \mathrm{~Hz}, 6-\mathrm{H}), 7.00(1 \mathrm{H}$, $\mathrm{d}, \mathrm{J}=2.4 \mathrm{~Hz}, 8-\mathrm{H}), 6.99(1 \mathrm{H}, \mathrm{d}, \mathrm{J}=2.4 \mathrm{~Hz}, 5-\mathrm{H}), 6.96\left(1 \mathrm{H}, \mathrm{dd}, \mathrm{J}=2.3 \mathrm{~Hz}, \mathrm{~J}=9.1 \mathrm{~Hz}, 4^{\prime}-\mathrm{H}\right), 6.23$ $(1 \mathrm{H}, \mathrm{d}, \mathrm{J}=8.5 \mathrm{~Hz}, 3-\mathrm{H}), 5.22\left(1 \mathrm{H}, \mathrm{s}, \mathrm{OCH}_{2}\right), 4.77\left(1 \mathrm{H}, \mathrm{s}, \mathrm{OCH}_{2}\right), 2.40\left(3 \mathrm{H}, \mathrm{s}, \mathrm{CH}_{3}\right) ; \mathrm{LC}-\mathrm{MS}: \mathrm{m} / \mathrm{z}$ $327(\mathrm{M}+1)$.

2-[(4-Methyl-2-oxo-2H-chromen-7-yl)oxy]-N'-[(thiophen-2-yl)methylene]acetohydrazide (4n). M.p. 274-276 ${ }^{\circ} \mathrm{C}$; IR (KBr) v: 3055, 2917, 1732, 1685, 1618, 1513, 1432, 1390, 1272 , $1143,1085 \mathrm{~cm}^{-1} ;{ }^{1} \mathrm{H}$ NMR (400 MHz, DMSO, ppm), $\delta=11.62(1 \mathrm{H}, \mathrm{d}, \mathrm{J}=21.8 \mathrm{~Hz}, \mathrm{HC}=\mathrm{N}), 8.31$ \& $8.20(1 \mathrm{H}, 2 \mathrm{~s}, \mathrm{NH}), 7.71(1 \mathrm{H}, \mathrm{d}, \mathrm{J}=5.8 \mathrm{~Hz}, 6-\mathrm{H}), 7.65\left(1 \mathrm{H}, \mathrm{d}, \mathrm{J}=8.0 \mathrm{~Hz}, 5^{\prime}-\mathrm{H}\right), 7.47\left(1 \mathrm{H}, \mathrm{s}, 3^{\prime}-\right.$ $\mathrm{H}), 7.13\left(1 \mathrm{H}, \mathrm{m}, 4^{\prime}-\mathrm{H}\right), 7.00(2 \mathrm{H}, \mathrm{m}, 5,8-\mathrm{H}), 6.23(1 \mathrm{H}, \mathrm{d}, \mathrm{J}=7.7 \mathrm{~Hz}, 3-\mathrm{H}), 5.20\left(1 \mathrm{H}, \mathrm{s}, \mathrm{OCH}_{2}\right)$, $4.79\left(1 \mathrm{H}, \mathrm{s}, \mathrm{OCH}_{2}\right), 2.40\left(3 \mathrm{H}, \mathrm{s}, \mathrm{CH}_{3}\right) ; \mathrm{LC}-\mathrm{MS}: \mathrm{m} / \mathrm{z} 341(\mathrm{M}-1)$.

2-[(4-Methyl-2-oxo-2H-chromen-7-yl)oxy]- $\boldsymbol{N}^{\prime}-[(\mathbf{1 H}$-imidazol-2-yl)methylene]acetohydrazide (4o). M.p. 256-258 ${ }^{\circ} \mathrm{C}$; IR (KBr) v: 3316, 3126, 3030, 2915, 1715, 1683, 1626, 1536, 1446, 1386, 1257, 1150, $1087 \mathrm{~cm}^{-1}$; ${ }^{1} \mathrm{H}$ NMR (400 MHz, DMSO, ppm), $\delta=12.60(1 \mathrm{H}, \mathrm{s}, \mathrm{NH}), 11.65$ $(1 \mathrm{H}, \mathrm{d}, \mathrm{J}=10.2 \mathrm{~Hz}, \mathrm{HC}=\mathrm{N}), 8.23 \& 7.89(1 \mathrm{H}, 2 \mathrm{~s}, \mathrm{NH}), 7.71(1 \mathrm{H}, \mathrm{d}, \mathrm{J}=3.4 \mathrm{~Hz}, 6-\mathrm{H}), 7.34(1 \mathrm{H}, \mathrm{s}$, 4'-H), 7.07 (1H, s, 5'-H), $7.03(1 \mathrm{H}, \mathrm{d}, \mathrm{J}=2.3 \mathrm{~Hz}, 8-\mathrm{H}), 7.00(1 \mathrm{H}, \mathrm{d}, \mathrm{J}=2.2 \mathrm{~Hz}, 5-\mathrm{H}), 6.23(1 \mathrm{H}, \mathrm{d}$, $\mathrm{J}=5.8 \mathrm{~Hz}, 3-\mathrm{H}), 5.26\left(1 \mathrm{H}, \mathrm{s}, \mathrm{OCH}_{2}\right), 4.82\left(1 \mathrm{H}, \mathrm{s}, \mathrm{OCH}_{2}\right), 2.40\left(3 \mathrm{H}, \mathrm{s}, \mathrm{CH}_{3}\right) ; \mathrm{LC}-\mathrm{MS}: \mathrm{m} / \mathrm{z} 327$ $(\mathrm{M}+1)$.

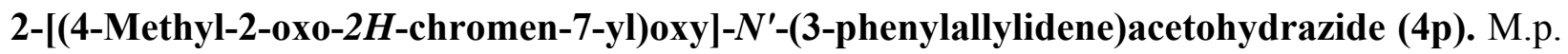
270-272 ${ }^{\circ} \mathrm{C}$; IR (KBr) v: 3313, 3043, 2914, 1704, 1685, 1625, 1533, 1421, 1390, 1251, 1151, $1084 \mathrm{~cm}^{-1} ;{ }^{1} \mathrm{H}$ NMR (400 MHz, DMSO, ppm), $\delta=11.54(1 \mathrm{H}, \mathrm{d}, \mathrm{J}=15.3 \mathrm{~Hz}, \mathrm{HC}=\mathrm{N}), 8.09 \&$ $7.84(1 \mathrm{H}, 2 \mathrm{~d}, \mathrm{~J}=8.8 \mathrm{~Hz}, \mathrm{NH}), 7.70(1 \mathrm{H}, \mathrm{d}, \mathrm{J}=8.8 \mathrm{~Hz}, 6-\mathrm{H}), 7.61\left(2 \mathrm{H}, \mathrm{d}, 2^{\prime}, 6^{\prime}-\mathrm{H}\right), 7.38(2 \mathrm{H}, \mathrm{t}, \mathrm{J}=$ 
7.2Hz, 4'-H), 7.09 (4H, m, 5, 8-H, HC=CH), $6.22(1 \mathrm{H}, \mathrm{d}, \mathrm{J}=7.8 \mathrm{~Hz}, 3-\mathrm{H}), 5.18\left(1 \mathrm{H}, \mathrm{s}, \mathrm{OCH}_{2}\right)$, $4.78\left(1 \mathrm{H}, \mathrm{s}, \mathrm{OCH}_{2}\right), 2.40\left(3 \mathrm{H}, \mathrm{s}, \mathrm{CH}_{3}\right)$; LC-MS: m/z $363(\mathrm{M}+1)$.

\section{Acknowledgements}

Authors, express thanks to the Administration of VIT University, Vellore for providing required facilities to support this work.

\section{References}

1. Abu-Hussen, A. A. A. J. Coord. Chem. 2006, 59, 157.

2. Sithambaram Karthikeyan, M.; Jagadesh Prasad, D.; Poojary, B.; Subramanya Bhat, K. Bioorg. Med. Chem. 2006, 14, 7482.

3. Singh, K.; Barwa, M. S.; Tyagi, P. Eur. J. Med. Chem. 2006, 41, 1.

4. Pannerselvam, P.; Nair, R. R.; Vijayalakshmi, G.; Subramanian, E. H.; Sridhar, S. K. Eur. J. Med. Chem. 2005, 40, 225.

5. Sridhar, S. K.; Saravan, M.; Ramesh, A. Eur. J. Med. Chem. 2001, 36, 615.

6. Pandeya, S. N.; Sriram, D.; Nath, G.; Declercq, E. Eur. J. Pharmacol. 1999, 9, 25.

7. Mladenova, R.; Ignatova, M.; Manolova, N.; Petrova, T.; Rashkov, I. Eur. Polym. J. 2002, 38, 989.

8. Walsh, O. M.; Meegan, M. J.; Prendergast, R. M.; Nakib, T. A. Eur. J. Med. Chem. 1996, 31, 989.

9. Arora, K.; Sharma, K. P. Synth. React. Inorg. Met.-Org. Chem. 2003, 32, 913.

10. Vigato, P. A.; Tamburini, S. Coord. Chem. Rev. 2004, 248, 1717.

11. Katsuki, T. Coord. Chem. Rev. 1995, 140, 189.

12. Thangadurai, T. D.; Gowri, M.; Natarajan, K. Synth. React. Inorg. Met.-Org. Chem. 2002, 32, 329.

13. Ramesh, R.; Sivagamasundari, M. Synth. React. Inorg. Met.-Org. Chem. 2003, 33, 899.

14. Schonberg, A.; Latif, N. J. Am. Chem. Soc. 1954, 76, 6208.

15. Mitra, A. K.; Misra, S. K.; Patra, A. Synth. Commun. 1980, 10, 915.

16. Singer, L. A.; Kong, N. P. J. Am. Chem. Soc. 1966, 88, 5213.

17. Jin, L.; Chen, J.; Song, B.; Chen, Z.; Yang, S.; Li, Q.; Hu, D.; Xu, R. Bioorg. Med. Chem. Lett. 2006, 16, 5036.

18. Miyano, M.; Clifford, R. D. J. Org. Chem. 1972, 37, 259.

19. Canpolat, E.; Kaya, M. Turk. J. Chem. 2005, 29, 409.

20. Tong-Shuang, L.; Zhan-Hui, Z.; Yang, F.; Cheng-Guang F. J. Chem. Res. (S) 1998, 38.

21. Khan, K. M.; Zafar, S. S. Nat. Prod. Res. 2004, 18, 21.

22. Agarwal, Y. K.; Manna, K.; Bhatt, H.; Gogai, P.; Babu, V. H.; Srinivasan, K. K. Ind. J. 
Heter. Chem. 2007, 16, 263.

23. Khanum, S. A.; Shashikanth, S.; Deepak, A. V. Bioorg. Chem. 2004, 32, 211.

24. Khan, M. S. Y.; Akhtar, M. Ind. J. Chem. 2003, 42B, 900. 